http://dx.doi.org/10.18778/2196-8403.2018.07

ELŻBIETA TOMASI-KAPRAL

\title{
Das Recht auf Vergessen? Zu konkurrierenden Modellen der Vergangenheitsaufarbeitung in Christoph Heins Glückskind mit Vater
}

Analysiert wird der Roman im Hinblick auf die Bezüge zur deutschen Geschichte und zu Modellen ihrer Aufarbeitung. Betont werden die Unterschiede zwischen der ost- und westdeutschen Geschichtspolitik nach 1945 und ihre Konsequenzen für die beiden Gesellschaften. Ersichtlich wird dadurch die wichtige Rolle der Literatur im Prozess der Herausbildung des kollektiven Gedächtnisses im Hinblick auf die traumatischen Aspekte der Vergangenheit.

The Right to Forget? Competing models of settling accounts with the past in Christoph Hein's "Happy Child with Father"

The article presents an analysis of various models of coping with recent German history as depicted in the novel. The author of the article draws attention to the differences between East German and West German historical policies after 1945 and the consequences of these policies for both German societies. She emphasises the important part literature plays in the process of creating a collective memory of an uncomfortable, and often traumatic, past.

Prawo do zapomnienia? O konkurujących ze sobą modelach rozliczenia z przeszłością w powieści Christopha Heina „Szczęśliwe dziecko z ojcem”

Artykuł proponuje analizę powieści pod kątem zawartych w niech odniesień do najnowszej historii Niemiec i modeli radzenia sobie z nią. Autorka zwraca uwagę na różnice we wschodnio- i zachodnioniemieckiej polityce historycznej po 1945 roku i konsekwencje, jakie z nich wynikały dla obu społeczeństw niemieckich. Podkreśla przy tym ważną rolę literatury w procesie tworzenia pamięci kolektywnej o niewygodnej, bo często traumatycznej przeszłości.

Die Zeit des Nationalsozialismus und des Zweiten Weltkriegs gehört in der zeitgenössischen deutschen Literatur wohl zu den am häufigsten thematisierten Aspekten der Vergangenheit. Auch beinahe 75 Jahre nach dem Ende des Zweiten 
Weltkrieges kann man immer noch nicht sagen, dass dies ein abgeschlossenes Kapitel der Geschichte ist. Die Ereignisse jener Zeit und der Umgang mit ihnen sorgen nach wie vor für starke Emotionen, die auf die nächsten Generationen übertragen werden. Der Nationalsozialismus kehrt immer wieder in die politischen Debatten zurück und ist somit im öffentlichen Diskurs fest verankert. Eine Erklärung für die in den letzten Jahrzehnten deutlich zunehmende Intensivierung dieses Diskurses ist unter anderem die Tatsache, dass es immer weniger AugenzeugInnen gibt, die einen Erfahrungsbezug zu der Zeit des Dritten Reichs hatten, was zur Folge hat, dass das kommunikative Gedächtnis in Bezug auf den Nationalsozialismus langsam schwindet und durch das kulturelle Gedächtnis ersetzt wird. In Bezug darauf formulierte WOLFRUM für das Ende des 20. Jahrhunderts die These, dass sich die deutsche Gesellschaft, ,in einem Gezeitenwechsel der Erinnerung" befindet (WOLFRUM 2002:144). Das kulturelle Gedächtnis basiert bekanntlich jedoch nicht mehr auf unmittelbaren, persönlichen Erfahrungen, sondern auf symbolischen Formen des Gedächtnisses, welche man RAIBLE zufolge als „Identifikationsangebote und Identifikationsmittel für die Mitglieder der Gemeinschaft" verstehen soll (RAIBLE 1991:315).

Auch die deutsche Wiedervereinigung von 1989/1990 bildete einen Auslöser für die erneute Beschäftigung mit der nationalsozialistischen Vergangenheit. Es begann die Zeit einer intensiven Auseinandersetzung mit solchen Themen wie Schuld, belastendes Erbe des Nationalsozialismus oder Vergangenheitsbewältigung. Die neue politische und gesellschaftliche Situation bedurfte einer Revision von bisher praktizierten Herangehensweisen an diese komplexe Problematik. Sowohl die west- als auch die ostdeutsche Erinnerungskultur mussten durch ein neues, diesmal gesamtdeutsches Modell der Erinnerung ersetzt werden (vgl. WOLFRUM 2002:346-356).

Eine wichtige Rolle spielt in diesem Kontext neben dem politischen und historischen auch der literarische Diskurs. Auf eine bedeutende Rolle der Literatur bei der Gestaltung des Gedächtnisses der jeweiligen Nation, Ethnie oder Gemeinschaft weisen in ihren wissenschaftlichen Studien neben ALEIDA ASSMANN (1999) unter anderem RENATE LACHMANN (1990) und ASTRID ERLL (2005) hin. JANUSZ GOLEC und IRMELA VON DER LÜHE betonen in der Einführung zu ihrem Sammelband Geschichte und Gedächtnis in der Literatur vom 18. bis 21. Jahrhundert, dass die aus dem politischen oder medialen Diskurs oft verdrängten Traumata und andere politisch unbequeme und deswegen tabuisierte Begebenheiten aus der Vergangenheit eben durch die Literatur ins Gedächtnis zurückgerufen werden können (vgl. GOLEC / VON DER LÜHE 2011:10). Eine so gelesene literarische Gedächtnisgeschichte ist demnach eine Art kritische Revision der 
Strategien, die in der Erinnerungspolitik der jeweiligen Gesellschaft verwendet werden. Sie kann ein wichtiges Werkzeug bei der Korrektur, Infragestellung oder sogar Negierung der Maßnahmen gesehen werden, die ergriffen wurden, um das kollektive Gedächtnis einer Nation zu instrumentalisieren.

\section{Ostdeutsche Erinnerungspolitik - Konflikt zwischen öffentlichem Gedenken und persönlichem Erinnern}

In Bezug auf die deutsche Nachkriegsliteratur muss zwischen der ost- und westdeutschen Literatur unterschieden werden, wo die unterschiedliche literarische Umsetzung des Nationalsozialismus durch die jeweilige Geschichtspolitik und Erinnerungskultur stark beeinflusst wurde. Während in der DDR der Prozess der offiziellen Vergangenheitsaufarbeitung gleich nach der Gründung dieses Staates in den ersten Nachkriegsjahren durch den von der Regierung verordneten Antifaschismus als beendet erklärt wurde, begann die Auseinandersetzung mit der nationalsozialistischen Vergangenheit in der BRD erst Mitte der 1960er Jahre, ausgelöst durch die Auschwitz-Prozesse und Protestwelle des Jahres 1968 (vgl. ASSMANN 2011:30).

In diesem Jahr ergriff in der BRD die Nachkriegsgeneration das Wort, welche sich selbst als eine die ungewollte Last des Nationalsozialismus tragende Generation sah, der Elterngeneration Passivität und mangelnden Widerstand gegen die nationalsozialistische Politik unterstellte und sie hiermit als schuldig für die in der Zeit des Zweiten Weltkriegs begangenen Verbrechen erklärte. Die sogenannte 68er-Generation wollte im Gegensatz zu ihren Eltern über die schwierige Vergangenheit sprechen und diese vor allem vor dem Vergessen bewahren, indem sie der massiven Amnesie eine offene Auseinandersetzung entgegensetzte. Sie waren auch bereit, die Schuld ihrer Eltern auf sich zu nehmen, anders als die Elterngeneration, die eher dazu tendierte, jegliche Schuld von sich zu weisen:

Die Übernahme der von den Eltern bestrittenen und abgespaltenen Schuld und ihre

Bearbeitung war die eine Seite der Erinnerungsmission der 68er Generation, die andere Seite war die Hinwendung zu den jüdischen Opfern, deren Zeugnisse sie anhörten und mit deren Geschichten und Kultur sie sich identifizierten. (ASSMANN 2006:170)

Doch es handelt sich hierbei um die westdeutsche Entwicklung, die in der DDR wohl kaum eine Entsprechung hatte. Die ostdeutschen Proteste des Jahres 1968 hatten einen anderen Charakter und richteten sich in erster Linie gegen die 
sowjetische Unterdrückung des Prager Frühlings und nicht gegen die schweigende Elterngeneration. Dies war unter anderem dadurch bedingt, dass die DDRRegierung eine durchaus andere Herangehensweise an die jüngste Geschichte Deutschlands entwickelte. Der proklamierte Antifaschismus bildete den Gründungsmythos des ostdeutschen Staates, und obwohl die ostdeutsche Politik in dieser Hinsicht viel zu wünschen übrig ließ, sollte man bedenken, dass der ,Hitlerfaschismus“ in den ersten Jahren nach der Gründung der DDR viel deutlicher und konsequenter angeprangert wurde, als dies in der BRD der Fall war. Besonders in den ersten Nachkriegsjahren hatten es die VertreterInnen der neuen Macht darauf abgesehen, jene Menschen aus dem öffentlichen Leben zu entfernen, die in das alte System verwickelt waren (vgl. GIESEKE 2010:82).

Nicht immer wurde jedoch der von der sowjetischen Besatzungsmacht angeordnete und von der DDR-Regierung übernommene Antifaschismus in die Tat umgesetzt, worauf heute immer häufiger die Geschichtsforschung hinweist. ${ }^{1}$ Besonders in den 1960er Jahren wurden die ehemaligen Nazis in der DDR zur Mitarbeit bei der Stasi gezwungen, indem man ihnen klar machte, dass dies der einzige Weg sei, die sie kompromittierende Nazivergangenheit abzubüßen und sich in den Augen der neuen Macht zu rehabilitieren. Die ehemaligen Nazis konnten für ihre Mitarbeit bei der Staatssicherheit und aktive Teilnahme am Aufbau des Sozialismus auf zahlreiche Gratifikationen und neue Karrieremöglichkeiten zählen (vgl. SCHULZ 2014). Der deklarierte Antifaschismus bildete einen wichtigen Bestandteil des ideologischen Krieges gegen Westdeutschland und ein wirksames Propagandamittel, das der Regierung die Loyalität des Volkes garantieren sollte. Es ist zu betonen, dass das offizielle Nachkriegsbekenntnis zur kommunistischen Ideologie und das willige Engagement für den Aufbau einer neuen politischen Wirklichkeit von vielen als ein Akt der Buße, ein gewisses Reinigungsritual des Gewissens betrachtet wurde, welches ermöglichte, sich von der problematischen Vergangenheit loszusprechen. Angesichts dessen wundert es nicht, dass in der DDR keine sachliche öffentliche Debatte über die nationalsozialistische Vergangenheit möglich war. Als einzig Schuldige an

Mit dieser Problematik befassen sich u. a. HenRy LeIDE (2007): NS-Verbrecher und Staatssicherheit. Die geheime Vergangenheitspolitik der DDR und ANETTE WeINKE (2002): Die Verfolgung von Nazi-Tätern im geteilten Deutschland. Vergangenheitsbewältigungen 1949-1969 oder eine deutsch-deutsche Beziehungsgeschichte im Kalten Krieg. 
den damals begangenen Verbrechen galt offiziell die Bundesrepublik Deutschland, was der ostdeutschen Regierung ermöglichte, jedes Erbe des Nationalsozialismus auszuschlagen (vgl. MÜNKLER 2009:43).

Eine wichtige Rolle bei der Verbreitung dieser Auslegungsart der Geschichte kam in der ostdeutschen Gesellschaft der Literatur zu. Die Abrechnungsproblematik wurde in der ostdeutschen Literatur schon Ende 1940er Jahre aufgegriffen. Die damals publizierten, auf persönlichen Erlebnissen der SchriftstellerInnen (manche waren bei der Wehrmacht und manche Häftlinge in Konzentrationslagern) basierenden Romane hatten einen durchaus autobiographischen Charakter und wurden nach von der DDR-Regierung erarbeiteten Richtlinien geschrieben. Ihnen zufolge sollte die antifaschistische ostdeutsche Literatur in erster Linie der Erziehung der neuen Gesellschaft dienen, indem sie den LeserInnen konkrete, nachahmungswürdige Verhaltensmuster lieferte. In diesen Texten dominierten zwei Tendenzen: Heroisierung des kommunistischen Widerstands im Nationalsozialismus und Erschaffung von Figuren, die infolge einer inneren Wandlung noch während des Krieges zur Besinnung kamen und zu überzeugten KommunistInnen wurden. Schon Ende der 1950er Jahre wurden jedoch im ostdeutschen literarischen Milieu kritische Stimmen hörbar, die darauf aufmerksam machten, dass solch eine Darstellung der nicht so fern liegenden Vergangenheit, welche nichtsdestotrotz im individuellen Gedächtnis von durchschnittlichen DDR-BürgerInnen vorhanden war, zu realitätsfremd war, als dass man sich mit ihr hätte identifizieren können. Die ostdeutschen LeserInnen hatten durchaus andere Erfahrungen aus dieser Zeit - die meisten waren in den Kriegsjahren weder beim kommunistischen Widerstand tätig noch bereit, die nationalsozialistische Ideologie in Frage zu stellen, wie dies Romanfiguren taten. Deswegen postulierten viele SchriftstellerInnen (darunter Christa Wolf, Erich Loest, Hermann Kant, Frank Wagner), dass die Figuren der literarischen Werke nicht KommunistInnen, sondern eben Nazis sein sollten und dass die diesem Themenkomplex gewidmeten Romane die ganze Vielschichtigkeit des Prozesses der eventuell erfolgten inneren Wandlung bewusst machen sollten (RÜTHER 1991:145).

Eine in diesem Kontext wichtige Stimme ist die von Christoph Hein. Der 1944 im heutigen Niederschlesien geborene Schriftsteller zog nach dem Zweiten Weltkrieg mit seiner Familie in die DDR, wo er seit 1979 literarische Texte verfasste und veröffentlichte. Aufgrund der darin behandelten Thematik befanden sich beinahe alle Werke von Hein im Visier der ostdeutschen Zensur. Hein gehörte zu jenen DDR-SchriftstellerInnen, die in der Literatur eine Mög- 
lichkeit sahen, wichtige und kontroverse Aspekte des Lebens im real existierenden Sozialismus zur Sprache zu bringen, welche im offiziellen medialen und politischen, durch die Propaganda geprägten Diskurs tabuisiert wurden.

Christoph Hein äußerte sich mehrmals zu Fragen der ostdeutschen Kultur- und Geschichtspolitik. Zu seinen bekanntesten und meistzitierten Aussagen, welche seine Kritik an dem angeordneten Modell der Geschichtsdeutung beinhaltet, gehört seine Rede vom 14. September 1989 im Ostberliner Schriftstellerverband. In dieser später unter dem Titel Die fünfte Grundrechenart publizierten Rede kritisiert er den Bildungsprozess des ostdeutschen historischen Gedächtnisses mit folgenden Worten:

Die fünfte Grundrechenart besteht darin, dass zuerst der Schluss gezogen und das erforderliche und gewünschte Ergebnis darunter geschrieben wird. Das gibt dann einen festen Halt für die waghalsigen Operationen, die anschließend und über dem Schlussstrich erfolgen. [...] Unter dem Schlussstrich unserer, uns aus Schule und Zeitungen sattsam bekannten Geschichtsbetrachtung, unter dem Schlussstrich, über den sich dann das als wissenschaftlich, objektiv und gesetzmäßig bezeichnete Gebäude von Fakten, Folgerungen und Bewertungen aufbaut, um den endgültigen und bereits zuvor gezogenen Schluss zu beweisen, stand und steht das kräftige Wort vom „Sieger der Geschichte“. (HeIN 1989)

Hein wirft solch einer Sichtweise der Geschichte Absurdität und Verlogenheit vor und verlangt eine offene, konstruktive und sachliche Diskussion, damit eine faire Abrechnung mit der Vergangenheit erfolgen könnte, die auch jene Aspekte berücksichtigen würde, die bisher tabuisiert wurden. Die von Hein postulierte Revision der ostdeutschen Erinnerungspolitik erfolgte tatsächlich nach der Wende des Jahres 1989. Diese politische Wende hatte nämlich auch eine Wende in der literarischen Umsetzung der ,braunen Vergangenheit ${ }^{\text {‘ }}$ gebracht. Dies manifestierte sich unter anderem durch die erneute Welle der Väterbücher im ersten Jahrzehnt des 21. Jahrhunderts. 


\section{Glückskind mit Vater - (k)ein Vaterroman ${ }^{2}$ ?}

Väterbücher sind in der deutschsprachigen Literatur seit den 1970er Jahren präsent. Die Väterbücher der ersten Publikationswelle können als Folge des Generationenkonflikts von 1968 gelesen werden. Ihren Höhepunkt erreichte die Väterliteratur in den Jahren 1975 bis $1985 .{ }^{3}$ Man muss dabei jedoch anmerken, dass es sich damals vorwiegend um eine westdeutsche Entwicklung handelte. Den thematischen Schwerpunkt dieser Prosa bildete eine persönliche Auseinandersetzung der Kinder mit der Tatsache, dass ihre Eltern (vorwiegend Väter) in den Nationalsozialismus verstrickt waren. Als Auslöser dieser Auseinandersetzung dient in diesen Narrativen oft der Tod des Vaters und das dadurch entstandene Gefühl, das versäumte Gespräch nachholen zu müssen, weswegen auf das Medium Literatur zurückgegriffen wurde. Da die Vaterfigur zu diesem Zeitpunkt jedoch bereits schon fehlte, wurden imaginierte Gespräche bzw. Monologe geführt, die von einem vorwurfsvollen Ton geprägt sind und in den meisten Fällen einer Anklage ähneln (vgl. SCHULZE 2015:15).

2 In der Literaturwissenschaft haben sich mittlerweile solche Begriffe wie ,Väterliteratur', ,Väterromane' bzw. ,Väterbücher' etabliert, welche in Bezug auf die literarischen Texte verwendet werden, die dem facettenreichen Themenkomplex der Abrechnung mit der nationalsozialistischen Vergangenheit des Vaters (bzw. der ganzen Familie) gewidmet sind. Auch im vorliegenden Beitrag werden diese Begriffe verwendet, wobei sich die Autorin dessen bewusst ist, dass sie oft verwirrend und unpräzise sind, was aus der Heterogenität der dem Korpus Väterliteratur zugeordneten Texte resultiert. Dieses terminologische Problem wird unter anderem von JULIAN REIDY in seiner Arbeit Vergessen, was Eltern sind. Relektüre und literaturgeschichtliche Neusituierung der angeblichen Väterliteratur (2012) behandelt. Um der begrifflichen Verwirrung aus dem Wege zu gehen, wurde daher an einer weiteren Stelle des Beitrags auf die These von ALEIDA ASSMANN rekurriert, deren Idee, die zwei Wellen der Erinnerungsliteratur begrifflich voneinander abzugrenzen, eine zumindest partielle Ordnung in dieser Begriffsvielfalt schafft (vgl. AssMANN 2006:26).

3 Die bekanntesten Vaterromane, die im genannten Zeitraum im deutschsprachigen Raum veröffentlicht wurden, sind u. a. PETER HENISCHS Die kleine Figur meines Vaters (1975), BERnWARD VESPERS Die Reise (1977), CHRISTOPH MECKELS Suchbild. Über meinen Vater (1980), PETER HÄRTLINGS Nachgetragene Liebe (1980), NiKLAS FRANKS Der Vater. Eine Abrechnung (1987). 
Für die Väterbücher der zweiten Publikationswelle, die nach der Jahrtausendwende erfolgte ${ }^{4}$, ist dagegen das Motiv der Vater-Spuren-Suche charakteristisch (vgl. BOROWICZ 2013:15) und das Bemühen, den bisher verschwiegenen Teil der Familiengeschichte zu rekonstruieren. Es wird dabei nicht lediglich das kommunikative Gedächtnis (ebenfalls Familiengedächtnis) in Betracht gezogen, sondern es werden auch in den Archiven gefundene Bilder und Dokumente berücksichtigt. Darin wird auch das Bestreben der AutorInnen nach Authentizität sichtbar. Darüber hinaus wird die auf diese Art und Weise unternommene Auseinandersetzung mit der Vaterfigur auch als Versuch der Akzeptanz der eigenen Familiengeschichte und der nationalsozialistischen Vergangenheit schlechthin gedeutet (vgl. BOROWICZ 2013:17).

Um den unterschiedlichen Charakter der Texte dieser zwei Wellen der Erinnerungsliteratur zu betonen, erscheint es plausibel, der von ALEIDA ASSMANN unternommenen Unterscheidung zu folgen, die die Väterbücher der 1970er und 1980er Jahre als „Väterliteratur" und die nach der Jahrtausendwende publizierten Texte als „Familienromane“ bezeichnet (vgl. AsSMANN 2006:26). Wobei das, was diese Prosa verbindet, ein im Zentrum stehendes, ,autobiographisches oder fiktives Ich [ist, E.K.], das sich seiner Identität gegenüber der eigenen Familie und der deutschen Geschichte vergewissert" (ASSMANN 2006:26).

Als Genre wurde der Familienroman auch von den in der DDR sozialisierten AutorInnen, zu welchen Christoph Hein gehört, wiederentdeckt. Nachdem das in der DDR-Erinnerungspolitik geltende Antifaschismus- und Widerstandsnarrativ durch die Wende des Jahres 1989 entkräftet wurde, konnten andere, bisher marginalisierte Aspekte der nationalsozialistischen Vergangenheit wie traumatische Kriegserlebnisse, Holocaust oder Täterschaft der Eltern-Generation literarisch umgesetzt werden. So werden in der ,postostdeutschen Familienerinnerungsliteratur" (OSTHEIMER 2013:139) Motive wie die Nachwirkungen der NS-Zeit und ihre Folgen für die Nachfolgegenerationen aufgegriffen. Es wird auch die Frage gestellt, ob die belastende Vergangenheit transgenerationell

$4 \quad$ Als Beispiel könnten hier stellvertretend Uwe Tiмm Am Beispiel meines Bruders (2003), WibKe Brunns Meines Vaters Land. Geschichte einer deutschen Familie (2004), MARTIN Pollack Der Tote im Bunker. Bericht über meinen Vater (2004), Hans Weiss Mein Vater, der Krieg und ich (2005), Beate Niemanns Mein guter Vater. Mein Leben mit seiner Vergangenheit. Eine Täter-Biographie (2005) genannt werden. 
übermittelt wird und ob die Täterkinder die Schuld ihrer Eltern verantworten sollen (OSTHEIMER 2013:12).

Der Roman Glückskind mit Vater kann im Kontext solcher Familienromane gelesen werden. Er weist nämlich einige für dieses Genre typische Merkmale auf. Im Fall von Konstantin Boggosch, der Hauptfigur des Romans, handelt es sich in der Tat um ein Täterkind, das sich mit dem Vater(schatten) auseinandersetzt. Mittels dieser Auseinandersetzung mit der eigenen Familiengeschichte soll auch die Identität dieses fiktiven Ichs konstruiert werden. Die Handlung dieses Romans spielt sich größtenteils in der DDR ab und der thematische Schwerpunkt liegt auf dem Umgang des Protagonisten, aber auch der ostdeutschen Gesellschaft, mit dem Erbe des Nationalsozialismus. So werden im Roman Glückskind mit Vater verschiedene Gedächtnismodelle gegenübergestellt, allen voran das kollektive und das individuelle Gedächtnis, aber auch das Gedächtnis der Väter- und der Söhne-Generation. Es werden auch die Erinnerungspolitiken der beiden deutschen Staaten miteinander konfrontiert und dadurch verschiedene Auslegungsmodelle der Geschichte präsentiert. Im Hinblick darauf leistet der Roman von Christoph Hein einen wichtigen Beitrag zur Diskussion über die in beiden deutschen Staaten nach dem Zweiten Weltkrieg ausgearbeiteten Modelle der Erinnerungskultur, wobei man jedoch anmerken muss, dass die ostdeutsche Perspektive im Roman deutlich überwiegt.

In die im Roman Glückskind mit Vater präsentierten Familiengeschichte sind die wichtigsten Aspekte und Meilensteine der neuesten Geschichte Deutschlands eingeschrieben: der in den 1930er Jahren immer lauter werdende Nationalsozialismus, der Zweite Weltkrieg, der Holocaust, die Teilung Deutschlands, der Bau der Berliner Mauer, das Leben in der ostdeutschen, durch die sozialistische Diktatur geprägten Wirklichkeit, der Fall der Mauer und die daraus resultierte Vereinigung beider deutscher Staaten. Durch die im Roman dargestellte Welt wird man - abgesehen vom kurzen, einführenden Teil des Romans und Epilog - vom Ich-Erzähler Konstantin Boggosch geführt. Die im Roman dargestellte Geschichte wird also von einem autodiegetischen Erzähler präsentiert. Diese stark subjektive Erzählweise hat zur Folge, dass der Eindruck von Authentizität der Geschichte hervorgerufen wird. In der Tat wird der Roman von der Literaturkritik als „Deutschlandroman“ bzw. „Deutschlandchronik“ (BUß 2016) und die Begebenheiten aus dem Leben des Protagonisten als „eine deutsche Biographie“ (HILGRUBER 2016) bezeichnet. Es sollte jedoch darauf hingewiesen werden, dass Hein bei dieser Strategie der Authentizitätserzeugung nicht kohärent bleibt. Die dem Roman vorangestellte Information, dass „der hier erzählten Geschichte authentische Vorkommnisse zugrunde [liegen]“ 
und „die Personen der Handlung nicht frei erfunden [sind]“ (HEIN 2016:4) lassen tatsächlich vermuten, dass es sich um eine, wahre Geschichte' handelt. Genauso sind die im Roman genannten Ereignisse (z. B. Bau der Berliner Mauer), Personen (Heinrich Himmler und dessen Bruder Gebhard) und Orte (Marseille, Leipzig, Berlin, München, Buna-Werke), den LeserInnen historisch verbürgt. Gleichzeitig aber werden wichtige Informationen verschleiert wie der Heimatort des Protagonisten, der im Roman lediglich als G. bezeichnet wird. Auf diese Weise wird die Fiktion nur zum Teil durch die historische Faktizität legitimiert, durch welche beim Lesen Wiedererkennungseffekte eingesetzt werden (vgl. GANSEL 2006:53).

Konstantin Boggosch ist das im Titel genannte ,Glückskind', das jedoch auf Schritt und Tritt vom Schatten seines Vaters, des Nationalsozialisten Gerhard Müller, eingeholt wird. Die Geschichte des Vaters ist mit der des Sohnes aufs Engste verflochten. Gerhard Müller ist eine kontroverse Figur, die unterschiedliche Reaktionen und Urteile provoziert. Mit dieser Figur liefert Hein das Bild eines überzeugten Nationalsozialisten, welcher, der Idee der Vernichtung durch Arbeit huldigend, ein Konzentrationslager in unmittelbarer Nachbarschaft seiner, Vulcano-Werke' gründen wollte, um auf diese Art und Weise auf billige Arbeitskräfte für seine Fabrik zugreifen zu können. Für die lokale Bevölkerung der Stadt G., in welcher er mit seiner Familie lebte, galt er jedoch in erster Linie als hochgeschätzter Unternehmer, der wichtigste Arbeitgeber in der Region, der vielen Familien in schwierigen Zeiten eine sichere Arbeitsstelle und festes Einkommen garantierte. Seine Kontakte mit der politischen Elite des Dritten Reichs sorgten für Bewunderung und Respekt ihm gegenüber, was sich unter anderem darin manifestierte, dass ihm der Titel des Ehrenbürgers der Stadt G. verliehen wurde (vgl. HEIN 2016:91). Das Jahr 1945 brachte jedoch eine Kehrtwende und eine diametrale Änderung der Beurteilung von Gerhard Müller. Aus der Siegerperspektive - in diesem Fall handelte es sich um die Sowjetunion - war er in erster Linie ein Kriegsverbrecher, wovon seine zahlreichen, an der Ostfront und in den Konzentrationslagern auf den Gebieten des heutigen Polen begangenen Verbrechen zeugten. Für die meisten Bewohner der Stadt G., seine Frau inklusive, war dies ein durchaus neues, bisher unbekanntes und daher schwer akzeptables Gesicht Gerhard Müllers. Im Gespräch mit ihren Söhnen gab sie zu:

Mein Mann, euer Vater, hat Blut an den Händen. Er ist ein Verbrecher. Irgendwann hatte ich es begriffen. Ich habe mich lange dagegen gewehrt. Ich wollte, nein, ich konnte es nicht glauben, dass der Mann, in den ich einmal verliebt war, den ich geheiratet habe, der der Vater meiner Kinder ist und mit dem ich gemeinsam in einer Wohnung gelebt habe, dass dieser Mann ein Mörder ist, ein besonders brutaler und grauenvoller Verbrecher. Ein Kriegsverbrecher. (HEIN 2016:76) 
Auch die lokale Bevölkerung musste sich mit dieser neuen Wirklichkeit abfinden. Im offiziellen Diskurs galt Gerhard Müller zwar als ein Nazi-Verbrecher, was jedoch nicht bedeutete, dass alle, den Anweisungen der neuen Machthaber folgend, ihr bisheriges Bild des Protagonisten tatsächlich revidiert haben. Obwohl man in G. nicht gerne über Gerhard Müller sprach (vgl. HEIN 2016:54), konnte man deutlich erkennen, dass er ,auch noch nach seinem Tod und nach Kriegsende von einigen Mitbürgern geachtet [wurde], während viele ihn hassten und über ihn schimpften“ (HEIN 2016:55). Diese Janusköpfigkeit in der Beurteilung des Protagonisten ist auch unter den Familienmitgliedern sichtbar. Müllers älterer Sohn, der unter dem Einfluss des in München lebenden Onkels steht, lehnt die neue Wahrheit über seinen Vater entschieden ab, da sie für ihn lediglich eine unglaubwürdige russische Nachkriegspropaganda ist:

Das ist alles Russenpropaganda. Die Russen sind die Sieger, die sind die Besatzungsmacht, da gilt nur, was sie sagen. Aber das ist nicht wahr. Warum soll ich glauben, was die Russen und Polen über meinen Vater erzählen? Der hat schließlich gegen sie gekämpft, da erzählen sie jetzt Gräuelmärchen. (HEIN 2016:91)

In seinen Augen bleibt der Vater ,ein Kriegsheld, der für sein Land ehrenvoll gekämpft und sein Leben eingesetzt hatte, ein Soldat, der seine vaterländische Pflicht erfüllte, und kein verbrecherischer Nazi.“ (HEIN 2016:93) Eine durchaus andere Meinung repräsentiert in dieser Hinsicht die Ehefrau Müllers, Erika (die nach dem Krieg ihren Nachnamen und den ihrer beiden Kinder ändern ließ, indem sie zu ihrem Geburtsnamen Boggosch zurückkehrte) und ihr jüngerer Sohn Konstantin. Die von diesen beiden Figuren getroffenen Entscheidungen und Maßnahmen hatten zum Ziel, sich von der auf ihrer Familie lastenden Schuld zu befreien und jegliche Spuren zu verwischen, die auf ihre Beziehung zum Kriegsverbrecher hätten hinweisen können. Der Roman ist somit durch den ständigen Kampf zwischen Erinnerung und Vergessen, zwischen dem kollektiven Gedächtnis einer Gesellschaft und dem Individuum, das für sich das Recht aufs Vergessen beansprucht, dominiert. Durch den Roman geistert die Frage danach, ob ein Täterkind das Recht aufs Vergessen hat.

Das Erinnern und das Vergessen (bzw. Verdrängen) bilden wichtige Aspekte des Umgangs mit der Vergangenheit und des kulturellen Gedächtnisses und sind als solche miteinander untrennbar verbunden. Auf diese Verschränkung von Erinnern und Vergessen macht unter anderem ALEIDA ASSMANN (2006) aufmerksam. In der von ihr durchgeführten Analyse des individuellen und kollektiven Gedächtnisses der Deutschen nach 1945 unterscheidet sie zwischen fünf Strategien des Vergessens, welche sie in ihrer Publikation Der lange 
Schatten der Vergangenheit (ASSMANN 2006:169-182) erörtert. Auf Grundlage der von ALEIDA ASSMANN definierten Modelle des Vergessens der unbequemen Vergangenheit lässt sich die These aufstellen, dass es sich bei Heins Roman quasi um deren literarische Paraphrase handelt. Erika Boggosch, welche im Roman die ältere Generation der Augenzeugen vertritt, wählte anfangs die Strategie des Schweigens (vgl. ASSMANN 2006:176), da sie fest davon überzeugt war, dass dies der richtige Weg gewesen ist, um ihre Söhne vor der Last der Vergangenheit zu befreien:

Ich hatte das Gefühl, diese Vergangenheit sei endgültig tot und vorbei, um euch nicht das Leben schwer zu machen, wollte ich euch nichts erzählen. Ich wollte euch davor bewahren. Vor diesem Dreck. Vor dieser Schuld, an der ihr keinen Anteil habt, nicht den geringsten. (HEIN 2016:78)

Die schwierige Wahrheit wird erst zehn Jahre nach dem Krieg erzählt, wenn die Mutter von den beiden Söhnen zur Rede gestellt wird (vgl. HeIN 2016:53).

Die Haltung ihres älteren Sohnes und dessen Onkels, die nicht nur die Schuld Gerhard Müllers bezweifeln, sondern dessen Taten während des Krieges sogar glorifizieren und in seinem Tod Züge eines Martyriums sehen, kann man dagegen als Strategie des Umfälschens bezeichnen, welche ASSMANN wie folgt definiert:

Die Umfälschung zum Passenden vollzieht sich unter dem Druck des neuen Gedächtnisrahmens, indem aus kompromittierenden Familienmitgliedern moralische Lichtgestalten werden. Besonders markant tritt dieser Befund dort zutage, wo im Familiengespräch ausnahmsweise doch einmal von Schuld in Form von Morden und Erschießungen die Rede ist. (ASSMANN 2006:180)

Daraus wird ersichtlich, dass im Roman im Rahmen des Familiengedächtnisses zwei Extreme veranschaulicht werden: die anfangs von Erika Boggosch vorgenommene Tabuisierung ${ }^{5}$ einerseits und die Mythosbildung, für welche der Bruder von Gerhard Müller verantwortlich ist, andererseits.

Interessant ist in diesem Kontext auch die von Konstantin Boggosch repräsentierte Einstellung seiner Familiengeschichte gegenüber. Ähnlich wie sein Bruder vertritt er im Roman die sogenannte Generation der Söhne, jedoch ist seine Beziehung zum Vater (den er nota bene gar nicht kennenlernte, da dieser einige Monate vor seiner Geburt in Polen erhängt worden war) anfangs durch Ablehnung und Hass geprägt. Konstantin klagt seinen Vater jedoch nicht an, der zeitliche Abstand des Protagonisten von der im Roman erzählten Geschichte bewirkt, dass er darüber reflektiert und sie letztendlich auch akzeptiert. Diese weist in ihrer Studie DoMinIKa Borowicz hin (vgl. BOrowicz 2015:16). 
Akzeptanz der Vaterfigur und der eigenen Familiengeschichte erfolgt stufenweise, wird aber bereits durch die Präposition ,mit' im Titel des Romans ironisch angekündigt.

Im Unterschied zu den Familienromanen, in welchen sich die Täterkinder gezielt auf die Suche nach dem Vater bzw. dessen Spuren begeben, haben wir es hier mit einer entgegengesetzten Entwicklung zu tun: Konstantin wird unfreiwillig immer wieder mit seinem Vater konfrontiert, dem er zu entkommen versucht. Der Schatten von Gerhard Müller holt ihn jedoch überall ein - sei es in Marseille, wohin er sich, ähnlich wie die deutschen MigrantInnen der 1930er Jahre, begibt, sei es in Leipzig, wo er nach seiner Rückkehr aus Frankreich auf einen Neuanfang hofft. Überall lauert die väterliche Vorgeschichte auf ihn.

Die in Glückskind mit Vater dargestellte Familiengeschichte widerspiegelt darüber hinaus die zuvor erwähnten Unterschiede zwischen dem ost- und westdeutschen Modell der Aufarbeitung der nationalsozialistischen Vergangenheit. Die Heimatstadt Gerhard Müllers befindet sich nach der Teilung Deutschlands auf dem Gebiet der sowjetischen Besatzungszone. Laut der dort herrschenden politischen Doktrin wurde Müller posthum zum Kriegsverbrecher erklärt, was wiederum zur Folge hatte, dass seine Familie nicht nur, wie auch andere Privatbesitzer, ihr ganzes Vermögen verlor, sondern auch anderen Repressionen ausgesetzt wurde - Konstantin darf nicht an den Schülerolympiaden teilnehmen, da er, obwohl sportlich viel begabter als seine MitschülerInnen, als Sohn eines Kriegsverbrechers nicht dessen würdig ist, seine Schule zu repräsentieren. Obwohl er gute Leistungen in der Schule hat, darf er das Gymnasium nicht besuchen, wodurch ihm der Weg zum Abitur versperrt bleibt. Auch wenn in seinem Fall von einer Erziehung im Geiste der nationalsozialistischen Ideologie nicht die Rede sein kann, werden ihm in der DDR alle Türen verschlossen, sobald herauskommt, dass er ein Nazi-Kind ist. Stellvertretend kann an dieser Stelle das Gespräch des Protagonisten mit dem Rektor der Filmakademie zitiert werden, der die Verweigerung des Studienplatzes mit folgendem Argument rechtfertigt:

Die antifaschistische Ordnung und das demokratische Grundprinzip sind die Pfeiler, auf denen all unsere Ausbildungsstätten gegründet sind [...] und für das Ministerium sei es [...] nicht vorstellbar, dass die DEFA die Produktion ihrer antifaschistischen Filme [...] dem Sohn eines Kriegsverbrechers überlässt. (HeIN 2016:389)

Auch die Mutter des Protagonisten ist durch diese Maßnahmen betroffen. So darf sie trotz entsprechender Ausbildung in keiner Schule unterrichten, da sie als eine Person, die mit der feindlichen Ideologie infiziert war, einen schlechten Einfluss auf die im sozialistischen Geiste erzogene Jugend haben könnte. 
In der BRD, wohin nach Kriegsende der Bruder von Gerhard Müller zieht, hätte seine Familie auf mehr Verständnis hoffen können. Laut dem rechtskräftigen Urteil des Münchner Gerichts wurde die von polnischen Soldaten in der polnischen Kleinstadt Kutno durchgeführte Exekution Gerhard Müllers als Mord am Offizier der deutschen Wehrmacht erklärt, der nun als Kriegsopfer gelten durfte. Durch diesen Status hätte seine Familie auf eine Rente zählen können, was eine wichtige Finanzquelle in der schwierigen Nachkriegszeit hätte sein können (vgl. HEIN 2016:79). Erika Müller will jedoch keine Sonderrechte, ihren Mann situiert sie nämlich entschieden und konsequent auf der Seite der Täter und nicht der Opfer des Zweiten Weltkrieges. Auch Konstantin lehnt entschieden all das ab, was ihn in einen Zusammenhang mit Gerhard Müller bringen könnte. Er schämt sich seiner Familiengeschichte und meidet jegliches Gespräch über den Nationalsozialismus. Nach seinem Vater gefragt, gibt er immer dieselbe auswendig gelernte Antwort: „Mein Vater ist tot. Er ist im Krieg geblieben." (HEIN 2016:137) und fügt dann gleich sich rechtfertigend hinzu: „Aber ich weiß nicht viel darüber. Mutter sprach nur wenig mit meinem Bruder und mir über unseren Vater." (HEIN 2016:201). Seine Haltung ist durch Angst vor der Konfrontation mit der Vergangenheit und den Wunsch, sie aus dem Gedächtnis zu verdrängen, geprägt. Symbolisch wird das im Roman durch den zuvor erwähnten Namenswechsel veranschaulicht.

Es stellt sich jedoch heraus, dass alle Bemühungen von Erika und Konstantin Boggosch, sich vom Schatten Gerhard Müllers zu befreien, erfolglos bleiben. Sie erleiden eine Niederlage unter anderem in der Konfrontation mit dem institutionalisierten Gedächtnis, in welchem zahlreiche Dokumente gespeichert sind, welche auf der Vergangenheit ihrer Familie lasten. Das institutionalisierte Gedächtnis wird im Roman in erster Linie durch Archive und die dort gesammelten Akten repräsentiert, ist aber auch durch Behörden, BeamtInnen wie auch deren Schreiben vertreten. Während die nach dem Kriegsende eine neue Ordnung schaffende Gesellschaft darum bemüht ist, alle Repräsentationen der heiklen Vergangenheit aus dem öffentlichen Raum zu entfernen, sehen es die VertreterInnen der neuen Ordnung darauf ab, eben diese Vergangenheit in den Personalakten sorgsam aufzubewahren, wobei man besonders auf jene Tatsachen Wert legt, die von der Verwicklung des Einzelnen in das vergangene, als verbrecherisch erklärte System zeugen. Die Akten von Konstantin Boggosch ähneln einem Lagerraum, in welchem das Gedächtnis seiner Familie aufbewahrt ist. In mehreren Exemplaren stehen sie jeder Zeit der neuen Regierung zur Verfügung, die somit Kontrolle über betreffende Personen ausübt. Eine solche Kontrolle über die im Archiv aufbewahrten Akten ist gleichzeitig eine 
Kontrolle über das Gedächtnis, was ALEIDA ASSMANN mit folgenden Worten zum Ausdruck bringt:

Vor dem Archiv als Gedächtnis der Historie kommt allerdings das Archiv als Gedächtnis der Herrschaft. Dieses besteht aus Legaten und Testaten, aus Urkunden, die Beweischarakter haben für Ansprüche auf Macht, Besitz und Abstammung. [...] Kontrolle des Archivs ist Kontrolle des Gedächtnisses. [...] Dokumente, die rechtlich entwertet waren, wurden jedoch nicht vernichtet, sondern im Gegenteil gesammelt. (ASSMANN 1999:343-344)

Jedes Mal, wenn im Roman die Akten von Konstantin Boggosch auftauchen, bedeutet dies für den Ich-Erzähler eine schmerzhafte Konfrontation mit seiner hierin konservierten Familiengeschichte, welche ihn verfolgt und welche er loswerden will. Obwohl der Name des Protagonisten von seiner Mutter geändert wurde, wird er weiterhin in allen staatlichen Institutionen (unter anderem auch in der Schule) mit seinem alten Namen, also als Konstantin Müller, angesprochen (vgl. HeIN 2016:54). Dies betrifft auch die Zeit nach der Wiedervereinigung Deutschlands: In einem nach der Wende vom Finanzamt an ihn geschickten Brief wird er Konstantin Müller, Sohn Gerhard Müllers, genannt, da er behördlich immer noch unter diesem Namen registriert ist (vgl. HEIN 2016:30). Das Schreiben entlarvt somit plötzlich und unerwartet seine wahre, jahrelang selbst vor seiner Ehefrau und engsten Freunden versteckte Identität.

Eine in dieser Hinsicht besondere Funktion kommt im Roman den Antiquariaten zu. Auf seiner Flucht vor dem Vater gerät Konstantin Boggosch paradoxerweise an Orte, an denen dessen Geschichte aufbewahrt ist. Zweimal findet er Beschäftigung in Antiquariaten, die im Roman symbolisch für das ,Speichergedächtnis“ stehen, also für Orte, an welchen das aufbewahrt wird, „was von einer Gesellschaft zu einem bestimmten Zeitpunkt ausgeblendet, abgewiesen, ausgemustert oder verworfen wurde" (GANSEL 2006:54). Diese These findet ihre Bestätigung in folgender Aussage des französischen Antiquartiatsbesitzers Emanuel Dupreis in Bezug auf die Dokumentation seiner Widerstandsgruppe Combat de coqs 22 juin $^{6}$ : „Zwei Francs hat es nur gekostet, stell dir das vor.

$6 \quad$ Es handelt sich hierbei um eine Publikation, in welcher die Tätigkeit der französischen, studentischen Widerstandsgruppe, welcher die in Marseille getroffenen Freunde von Konstantin Boggosch angehörten, von ihrer Gründung am Anfang des zweiten Weltkrieges bis zum Zerfall nach dem Krieg dokumentiert wurde. Aus dem vierten Kapitel, das „Deutsche Lager“ betitelt ist und von der Gefangenschaft der Gruppenmitglieder in den Konzentrationslagern handelt, erfährt der Ich-Erzähler, dass sein Freund Duprais in demselben Konzentrationslager war, in 
Neu hat es zweitausend alte Francs gekostet, jetzt wird es für zwei verramscht. Keiner will mehr etwas davon wissen." (HEIN 2016:240)

An einer anderen Stelle des Romans wird jedoch darauf hingewiesen, dass die Aufgabe der Antiquariate nicht lediglich in dem Speichern von Ungewolltem, sondern vielmehr in dessen Aufbewahrung für die kommenden Generationen besteht: , $[\mathrm{H}]$ ier ist alles für die Ewigkeit festgehalten. Antiquare arbeiten für die Ewigkeiten, das gehört zum Beruf.“ (HEIN 2016:322)

So lässt Hein seinen Protagonisten, der sich vor der Geschichte seiner Familie befreien will und seinen Vater am liebsten aus seinem Gedächtnis löschen würde, ausgerechnet an Orten arbeiten, an welchen ihn diese Geschichte einholt und sorgfältig aufbewahrt wird.

Nicht nur die Akten des Protagonisten und die in den Antiquariaten momentan an acta gelegte Dokumentation Combat de coqs 22 juin bewahren seine Familiengeschichte auf. Sie bildet auch einen festen Bestandteil des Gedächtnisses der lokalen Bevölkerung der Stadt G., was in der letzten Szene des Romans, die schon im Nachwendedeutschland spielt, besonders deutlich wird, wenn der schon älter gewordene und pensionierte Ich-Erzähler beschließt, seine Heimatstadt zu besuchen, um sich dem ihn verfolgenden Schatten seines Vaters zu stellen und ihm vielleicht zum ersten Mal in seinem Leben furchtlos entgegenzutreten. Während seines Besuchs in der Stadt G. stellt sich jedoch heraus, dass er für die zufällig auf der Straße begegneten Bekannten immer noch Konstantin Müller ist; die Namen Müller und Boggosch werden in einem Atemzug genannt und verschmelzen zu einem Ganzen. Die Erinnerung an seinen Vater ist in der Stadt nach wie vor lebendig - die ihm einst gehörenden Häuser werden sorgfältig renoviert, auch seine Fabrik wird wieder instandgesetzt, sodass die Stadtbevölkerung dort wieder eine Beschäftigung finden kann. Aus der Aussage der Cousine des Protagonisten: „Hier heißt es einfach: Mein Mann hat Arbeit im KZ gefunden. Man denkt sich nichts dabei, es heißt halt so“ (HEIN 2016:521) geht deutlich hervor, dass trotz des zeitlichen Abstands die Erinnerung an die

welchem Gerhard Müller als Aufseher tätig war. Das letzte Kapitel der Publikation liefert darüber hinaus Informationen zum Tod von Gerhard Müller. In diesem Sinne bildet die Dokumentation Combat de coqs 22 juin eine wichtige Informationsquelle für Konstantin Boggosch, der anhand der dort versammelten Dokumente (Fotos, Zeugenberichte) das Bild seines Vaters, das bisher auf dem Familiengedächtnis basierte, um eine neue Dimension erweitern kann (vgl. HEIN 2016:231-232). 
NS-Zeit im Gedächtnis der lokalen Bevölkerung fest verankert ist. Die Versicherung, dass man sich dabei nichts denkt, klingt umso befremdlicher.

Die Rückkehr in seine Heimatstadt nach jahrzehntelanger Abwesenheit und die dortige Konfrontation mit seiner Vergangenheit veranschaulichen, dass Konstantin Boggosch seinen Kampf um das Recht aufs Vergessen eindeutig verliert. Trotz zahlreicher, oft riskanter Versuche, sich von seiner Vergangenheit zu befreien und die Figur des Vaters aus seinem Lebenslauf zu streichen, erweist er sich immer wieder als dessen Opfer.

Im Roman Glückskind mit Vater wird ein persönliches Zeugnis abgelegt. Der Ich-Erzähler lässt dabei sein ganzes Leben Revue passieren: Es werden seine Kindheitserlebnisse aus der unmittelbaren Nachkriegszeit wie auch wichtige Stationen aus der Jugendzeit und des Erwachsenenlebens in Erinnerung gerufen. Durch die Erzählung des Protagonisten über seine Bemühungen, der unbequemen Vergangenheit zu entkommen, wird im Roman gleichzeitig sein Erinnerungsprozess veranschaulicht. Im Fall von Konstantin Boggosch erfolgt, ähnlich wie in den Familienromanen, eine stufenweise (Re-)Konstruktion des Vaterbildes, worin jedoch keine explizite Absicht des Protagonisten liegt. Diese ungewollte (Re-)Konstruktion erfolgt weniger durch eigene Erinnerungen des Ich-Erzählers, sondern wird durch das kommunikative Gedächtnis und die ihm zugänglichen Medien wie Fotos, Briefe des Onkels und letztendlich die Dokumentation Combat de coqs 22 juin an ihn herangetragen.

Es sollte jedoch hervorgehoben werden, dass nicht das Erinnern, sondern der Wunsch nach Vergessen und Befreiung von der belastenden Geschichte den vom Protagonisten unternommenen Handlungen von Anfang an zugrunde liegt. Konstantin Boggosch, Sohn eines Kriegsverbrechers, will sich eine neue Identität schaffen, was durch die Namensänderung, Wohnortwechsel und eine konsequente Abkehr von der Familiengeschichte erreicht werden soll. In der von Hein konzipierten Romanwirklichkeit, welche durch eine „Diktatur der Erinnerung“ (WOLFF-POWĘSKA 2011:32) geprägt ist, wurde ihm jedoch das Recht aufs Vergessen konsequent abgesprochen. Das Ende des Romans veranschaulicht, dass der Protagonist sich mit diesem Sachverhalt abfindet und nachgibt. Der pensionierte Konstantin Boggosch weigert sich nicht mehr zu erinnern, sondern stellt sich seiner Vergangenheit und akzeptiert sie. In dieser Hinsicht erinnert der Roman Glückskind mit Vater an die für die DDR-Literatur charakteristischen sozialistischen Entwicklungsromane, in welchen der Protagonist den Weg zu sich selbst findet, indem er die Normen der sozialistischen Gesellschaft akzeptiert (vgl. GELBERG 2018:73). Der von Boggosch vollbrachte 
Akt der Auseinandersetzung mit seiner Familiengeschichte kann in diesem Sinne auch als Akzeptanz der konkreten gesellschaftlichen Wirklichkeit gedeutet werden, in der er mit der Zeit zu leben gelernt hat.

\section{Literatur}

Assmann, Aleida (1999): Erinnerungsräume. Formen und Wandlungen des kulturellen Gedächtnisses. München.

ASSMANN, AlEIDA (2006a): Der lange Schatten der Vergangenheit. Erinnerungskultur und Geschichtspolitik. München.

AsSMANN, AlEIDA (2006b): Generationsidentitäten und Vorurteilskulturen in der neuen deutschen Erinnerungsliteratur. Wien.

ASSMANN, AlEIDA (2011): Von kollektiver Gewalt zu gemeinsamer Zukunft. Vier Modelle für den Umgang mit traumatischer Vergangenheit. In: ASSMANN, ALEIDA/ ASSMANN, Wolfgang R. / Graf von Kalnein, Albert (eds.): Erinnerung und Gesellschaft. Formen der Aufarbeitung von Diktaturen in Europa. Berlin, 25-42.

Borowicz, Dominika (2013): Vater-Spuren-Suche. Auseinandersetzung mit der Vätergeneration in deutschsprachigen autobiographischen Texten von 1975 bis 2006. Göttingen.

Buß, Christian (2016): Deutschlandroman von Christoph Hein. Mein Vater, das Gespenst: https://www.spiegel.de/kultur/literatur/christoph-hein-glueckskind-mit-vater-meinvater-das-gespenst-a-1079585.html (05.06.2019).

ERLL, ASTRID (2005): Kollektives Gedächtnis und Erinnerungskulturen. Stuttgart/Weimar. GANSEL, CARSTEN (2006): Zwischen offiziellem Gedächtnis und Gegen-Erinnerung. Literatur und ,kollektives Gedächtnis ' in der DDR. In: Spiegel der Forschung 23:48-59.

Gelberg, Johanna M. (2018): Poetik und Politik der Grenze. Die Literatur der deutschdeutschen Teilung seit 1945. Bielefeld.

GIESEKE, JENS (2010): Antifaschistischer Staat und postfaschistische Gesellschaft: Die DDR, das MfS und die NS-Täter. In: Historical Social Research / Historische Sozialforschung 35:79-94.

GOLEC, JANUSZ / VON DER LÜHE, IRMELA (2011): Geschichte und Gedächtnis in der Literatur vom 18. bis 21. Jahrhundert. Frankfurt a.M.

HeIn, CHRISTOPH (1989): Die fünfte Grundrechenart. In: Die Zeit 41, online verfügbar: https://www.zeit.de/1989/41/die-fuenfte-grundrechenart/komplettansicht?print (10.02.2018).

HeIn, CHRistoph (2016): Glückskind mit Vater. Berlin.

HILGRUBER, KATRIN (2016): Christoph Hein: Glückskind mit Vater. Ein tragikomischer Lebensbericht: https://www.deutschlandfunk.de/christoph-hein-glueckskind-mit-vater-eintragikomischer.700.de.html?dram:article_id=365257 (05.06.2019).

LACHMANN, RENATE (1990): Gedächtnis und Literatur. Intertextualität in der russischen Moderne. Frankfurt a. M. 
LEIDE, HENRY (2007): NS-Verbrecher und Staatssicherheit. Die geheime Vergangenheitspolitik der DDR. Göttingen.

MüNKLER, Herfried (2009): Antifaschismus als Gründungsmythos der DDR, Abgrenzungsmechanismus nach Westen und Herrschaftsmittel nach Innen. In: Konrad Adenauer Stiftung (eds.): Antifaschismus als Staatsdoktrin der DDR. Berlin, 31-49.

OSTHEIMER, MichAEL (2013): Ungebetene Hinterlassenschaften. Zur literarischen Imagination über das familiäre Nachleben des Nationalsozialismus. Göttingen.

Raible, WolfGang (1991): Symbolische Formen, Medien, Identität. Tübingen.

REIDY, Julian (2012): Vergessen, was Eltern sind. Relektüre und literaturgeschichtliche Neusituierung der angeblichen Väterliteratur. Göttingen.

RÜTHER, GÜNTHER (1991): ,, Greif zur Feder, Kumpel. “Schriftsteller, Literatur und Politik in der DDR 1949-1990. Düsseldorf, 140-148.

SchulZ, BEnJAmin (2014): NS-Täter in der DDR. Wie die Stasi NS-Leute erpresste: https://www.spiegel.de/einestages/ns-taeter-in-der-ddr-wie-die-stasi-ss-leute-aus-auschwitz -erpresste-a-987462.html (20.05.2019).

SCHULZE, LINDA (2015): Aufarbeitung von nationalsozialistischer Vergangenheit in deutscher Gegenwartsliteratur. Hamburg.

Weinke, Anette (2002): Die Verfolgung von Nazi-Tätern im geteilten Deutschland. Vergangenheitsbewältigungen 1949-1969 oder eine deutsch-deutsche Beziehungsgeschichte im Kalten Krieg. Paderborn.

WolfF-PowęSKA, ANNA (2011): Pamięć, brzemię i uwolnienie. Niemcy wobec nazistowskiej przeszłości (1945-2010) [Erinnerung, Joch und Befreiung. Die Deutschen angesichts der nationalsozialistischen Vergangenheit (1945-2010)]. Poznań.

WOLFRUM, EDGAR (2002): Der Weg zur bundesrepublikanischen Erinnerung 1948-1990. In: Wolfrum, Edgar: Geschichtspolitik in der Bundesrepublik Deutschland. Darmstadt, 346-356.

Wolfrum, EdgAR (2002): Geschichte als Waffe. Vom Kaiserreich bis zur Wiedervereinigung. Göttingen. 